\title{
Stress myocardial blood flow correlates with ventricular function and synchrony better than myocardial perfusion reserve: A Nitrogen-13 ammonia PET study
}

Luis Eduardo Juárez-Orozco, MD, ${ }^{\mathrm{a}, \mathrm{b}, \mathrm{c}}$ Erick Alexanderson, $M D,{ }^{\mathrm{c}, \mathrm{j}}$ Rudi A. Dierckx, MD, PhD, ${ }^{\mathrm{a}}$ Hendrikus H. Boersma, PharmD, PhD, ${ }^{\mathrm{a}, \mathrm{g}}$ Johannes L. Hillege, MD, PhD, ${ }^{\mathrm{e}}$ Clark J. Zeebregts, MD, PhD, ${ }^{\mathrm{f}}$ Myriam M. Martínez-Aguilar, MD, ${ }^{\mathrm{b}, \mathrm{c}}$

Antonio Jordán-Ríos, MD, ${ }^{\text {h }}$ Ana Gabriela Ayala-German, MD, ${ }^{\text {b }}$ Niek Prakken, MD, PhD, ${ }^{a}$ Rene A. Tio, MD, PhD, ${ }^{\mathrm{d}}$ and Riemer H. Slart, $M D, \mathrm{PhD}^{\mathrm{a}, \mathrm{i}}$

a Department of Nuclear Medicine and Molecular Imaging, University of Groningen, University Medical Center Groningen, Groningen, The Netherlands

b PET/CT Unit, Faculty of Medicine, National Autonomous University of Mexico, Mexico City, Mexico

c Department of Nuclear Cardiology, Instituto Nacional de Cardiología "Ignacio Chávez", Mexico City, Mexico

d Department of Cardiology, University of Groningen, University Medical Center Groningen, Groningen, The Netherlands

e Department of Epidemiology, University of Groningen, University Medical Center Groningen, Groningen, The Netherlands

f Department of Surgery (Division of Vascular Surgery), University of Groningen, University Medical Center Groningen, Groningen, The Netherlands

g Department of Clinical Pharmacy and Pharmacology, University of Groningen, University Medical Center Groningen, Groningen, The Netherlands

h Mexican Faculty of Medicine, La Salle University, Mexico City, Mexico

i Department of Biomedical Photonic Imaging, University of Twente, Enschede, The Netherlands

i Department of Physiology, Faculty of Medicine, National Autonomous University of Mexico, Mexico City, Mexico

Received Jun 15, 2016; accepted Aug 16, 2016 doi: 10.1007/s12350-016-0669-y

Background. Cardiac PET quantifies stress myocardial blood flow (MBF) and perfusion reserve (MPR), while ECG-gated datasets can measure components of ventricular function simultaneously. Stress MBF seems to outperform MPR in the detection of significant CAD. However, it is uncertain which perfusion measurement is more related to ventricular function. We hypothesized that stress MBF correlates with ventricular function better than MPR in patients studied for suspected myocardial ischemia.

Methods. We studied 248 patients referred to a rest and adenosine-stress Nitrogen-13 ammonia PET. We performed a multivariate analysis using systolic function (left ventricular ejection fraction, LVEF), diastolic function (mean filling rate in diastole, MFR/3), and synchrony (Entropy) as the outcome variables, and stress MBF, MPR, and relevant covariates as the predictors. Secondarily, we repeated the analysis for the subgroup of patients with and without a previous myocardial infarction (MI).

Electronic supplementary material The online version of this article (doi:10.1007/s12350-016-0669-y) contains supplementary material, which is available to authorized users.

The authors of this article have provided a PowerPoint file, available for download at SpringerLink, which summarises the contents of the paper and is free for re-use at meetings and presentations. Search for the article DOI on SpringerLink.com.

Reprint requests: Erick Alexanderson, MD, Department of Nuclear Cardiology, Instituto Nacional de Cardiología "Ignacio Chávez", 
Results. 166 male and 82 female patients (mean age $63 \pm 11$ and $67 \pm 11$ year, respectively) were included. $60 \%$ of the patients presented hypertension, $57 \%$ dyslipidemia, $21 \%$ type 2 diabetes mellitus, $45 \%$ smoking, and $34.7 \%$ a previous MI. Mean stress MBF was $1.99 \pm 0.75$ $\mathrm{mL} / \mathrm{g} / \mathrm{min}, \mathrm{MPR}=2.55 \pm 0.89, \mathrm{LVEF}=61.6 \pm 15 \%, \mathrm{MFR} / 3=1.12 \pm 0.38 \mathrm{EDV} / \mathrm{s}$, and Entropy $=$ 45.6 $\pm 11.3 \%$. There was a significant correlation between stress MBF $(P<.001)$ and ventricular function. This was stronger than the one for MPR $(P=.063)$. Sex, age, diabetes, and extent of previous MI were also significant predictors. Results were similar for the analyses of the 2 subgroups.

Conclusion. Stress MBF is better correlated with ventricular function than MPR, as evaluated by Nitrogen-13 ammonia PET, independently from other relevant cardiovascular risk factors and clinical covariates. This relationship between coronary vasodilatory capacity and ventricular function is sustained across groups with and without a previous MI. (J Nucl Cardiol 2018;25:797-806.)

Key Words: PET • stress myocardial blood flow $\cdot$ myocardial perfusion reserve $\bullet$ ventricular function • coronary artery disease

\begin{tabular}{|ll|}
\hline Abbreviations \\
PET & Positron emission tomography \\
CAD & Coronary artery disease \\
MBF & Myocardial blood flow \\
MPR & Myocardial perfusion reserve \\
ECG & Electrocardiogram \\
MI & Myocardial infarction \\
LVEF & Left ventricular ejection fraction \\
MFR/3 & Mean filling rate during the first third \\
& of diastolic time \\
\hline
\end{tabular}

\section{See related editorial, pp. 807-808}

\section{INTRODUCTION}

Cardiac positron emission tomography (PET) imaging has developed as an important adjuvant for diagnostic, prognostic, and therapeutic evaluation in the evolving profile of coronary artery disease (CAD), and is currently the reference technique for the quantification of myocardial perfusion in absolute terms. ${ }^{1}$ This quantification of myocardial blood flow (MBF) is performed during rest and pharmacological stress, and the calculated ratio of stress MBF to rest MBF is known as the myocardial perfusion reserve (MPR). Stress MBF and MPR have shown superior diagnostic performance as compared to the evaluation of relative myocardial tracer retention and semiquantitative assessment. ${ }^{2-7}$

\footnotetext{
Juan Badiano \#1 Col. Sección XVI Del. Tlalpan, C.P 14080 Mexico City, Mexico; erickalexandersonmd@gmail.com 1071-3581/\$34.00

Copyright @ 2016 The Author(s). This article is published with open access at Springerlink.com
}

Recently, it has been suggested that stress MBF may perform better than MPR in the detection of flowlimiting CAD. This may be especially true in populations without previously known CAD or in conditions that show a higher rest MBF such as women, older patients, or those with arterial hypertension. ${ }^{8}$ Further, only a couple of studies have partially addressed the prognostic value of stress MBF over MPR for the occurrence of cardiovascular events. ${ }^{9,10}$

Furthermore, ECG-synchronized (gated) PET datasets can be simultaneously acquired offering the possibility to assess three areas of ventricular function, namely: systolic (i.e., ejection) and diastolic (i.e. filling) function, ${ }^{11-13}$ and ventricular synchrony (uniformity of left ventricular contraction). ${ }^{14,15}$ This is useful because cardiac function measurements can provide information for the evaluation of the effects of myocardial ischemia. ${ }^{16}$ Indeed, progressive hampering of ventricular function also constitutes an important component when addressing cardiovascular prognosis and risk stratification. As such, although quantitative measures of myocardial perfusion are predictive of coronary anatomic findings of CAD, it is still unknown whether stress MBF or MPR is better correlated with resulting ventricular function.

Therefore, the aim of the present study was to evaluate whether stress MBF or MPR, as measured by PET myocardial perfusion imaging, better correlates with ventricular function in the general population of patients with known or suspected CAD. Secondarily, we explored the relationship of stress MBF and MPR with ventricular function in patients with and without a previous myocardial infarction (MI) as it is known that there are differences in ventricular perfusion and function dynamics.

A supplementary analysis for subgroups of interest within patients without a previous MI (e.g. women and patients with arterial hypertension) was also conducted. 


\section{METHODS}

\section{Population}

A retrospective study was conducted including 248 patients referred to the PET/CT Unit at the National Autonomous University of Mexico in Mexico City by their cardiologist for the evaluation of suspected myocardial ischemia. Demographic and clinical data were retrieved through the electronic patient archive system. Several clinically relevant confounders were included in this study: sex, age, body mass index (BMI), hypertension, dyslipidemia, type 2 diabetes mellitus (DM), smoking habit, and semiquantitative perfusion scores (see ahead). Ethical approval was obtained for the conduction of the study from the local institutional ethics committee.

\section{PET Acquisition}

PET images were acquired on a whole-body 64-slice PET/CT scanner (Biograph True Point; Siemens Medical Solutions). PET data were acquired in 3-D list mode. Patients were studied after an overnight fast, and all refrained from caffeine-containing beverages or theophylline-containing medications for 24 hours before the study. Myocardial perfusion was assessed at rest and during vasodilator stress with adenosine and Nitrogen-13 ammonia as the perfusion radiotracer. Two CT-based transmission scans $(120 \mathrm{kVp}$; 20-30 mA; helical scan mode with a pitch of 1.35) were obtained before the rest perfusion studies and after the stress perfusion studies with normal breathing for correction of photon attenuation for PET. The registration of the CT attenuation map with the PET images was verified visually by an experienced technologist and alignment was corrected if necessary by manual 3-D translation. Regional myocardial perfusion was first assessed during rest using $740 \mathrm{MBq}$ of Nitrogen-13 ammonia. Rest imaging extended for 10 minutes and began a few seconds before the injection. The radiotracer was administered as a single peripheral intravenous bolus (3-5 seconds) followed by a $10-\mathrm{mL}$ saline flush. Thirty minutes later, a pharmacological stress test was performed, beginning with the injection of adenosine during a 6 -minute period $(140 \mathrm{mg} / \mathrm{kg}$ per minute). A second dose of $740 \mathrm{MBq}$ of Nitrogen-13 ammonia was injected at the third minute of the adenosine infusion. Stress images acquisition was started a few seconds before the radiotracer injection. Sixteen dynamic frames were reconstructed (twelve 10-s, two 30-s, one 1-minute, and one 6-minute frames, for a total of 10 minutes). Standard reconstruction (2-D attenuationweighted OSEM) was used with 3 iterations and 14 subsets and 3-D postfiltering with a 5-mm Gaussian kernel filter. Transverse data were reformatted to a $168 \times 168 \times 47$ matrix with $2 \mathrm{~mm}$ pixels for each dynamic frame.

\section{Perfusion Data Analysis}

\section{Semiquantitative Myocardial Perfu-}

sion. Images were interpreted semiquantitatively using the standard American Heart Association 5-point scoring system ${ }^{17}$ and traditional metrics were documented: summed difference score (SDS), summed stress score (SSS), and summed rest score (SRS). SRS (in a fixed perfusion defect) was considered as a measure of the extent and severity of a previous MI.

Quantitative Myocardial Perfusion. Left ventricular contours and input function regions were obtained automatically allowing minimal observer intervention in QPS software package (Cedars-Sinai, Los Angeles, CA, USA). Dynamic myocardial samples were obtained from the polar map by analyzing all time frames within the fixed left ventricular contour boundaries. Quantitative rest and stress MBF values ( $\mathrm{mL} / \mathrm{g} /$ minute) were computed for each sample on the polar map as described previously ${ }^{18}$ using a previously described 2-tissue compartment pharmacokinetic model for Nitrogen-13 ammonia. ${ }^{19}$ MPR was calculated as the ratio between stress MBF and rest MBF (making it a unitless variable). Rest MBF was corrected accordingly for the ratepressure product (RPP). ${ }^{20}$ The global MPR and stress MBF were calculated within the whole left ventricular region (as defined by the left ventricle long-axis plane) as parameters of interest for our analysis.

\section{Ventricular Function Data Analysis}

ECG-gated stress images were analyzed with the QGS software package (Cedars-Sinai, Los Angeles, CA, USA). ${ }^{21}$ Short-axis images were processed and ventricular edges and cavity volumes were calculated for each of the re-binned 8 dynamic frames that were reconstructed for the average cardiac cycle. The algorithm for determining edges and calculating volume has been described previously. ${ }^{22}$

Systolic function was evaluated through left ventricular ejection fraction (LVEF) (\% of the end-diastolic volume ejected) using the average time-volume curves. Next, diastolic function was evaluated with the mean filling rate during the first third of diastole (MFR/3) as a surrogate marker. MFR/3 was obtained from the first derivative of the left ventricular time-volume curve and expressed in end-diastolic volumes per second (EDV/s) as it is adjusted to the ventricle dimensions on a patient-by-patient basis. ${ }^{23,24}$

Finally, ventricular synchrony was evaluated through Entropy, which is a measurement of uniformity of the onset and progression of wall motion throughout the cardiac cycle. ${ }^{25}$ Entropy was expressed as a percentage (ranging from 0 to 100) with greater percentages reflecting a lesser uniformity of ventricular fiber contraction. ${ }^{14}$ It was obtained by phase analysis (embedded in the QGS software package [Research Edition, PET Processing plugin, Cedars-Sinai]). Entropy was used because it constitutes an expression of (dys)synchrony that is not dependent on phase similarity ${ }^{25}$ as other described parameters (Bandwidth and Standard Deviation).

\section{Statistical Analysis}

All continuous variables are described as mean \pm standard deviation, and categorical variables are expressed as frequencies with percentages. Between-group comparisons were made using independent samples $t$ tests. 
The biserial correlations (using Pearson's correlation coefficient) between LVEF, MFR/3, and Entropy were evaluated. Next, a general multivariate analysis of covariance (MANCOVA) was performed including sex, age, BMI, hypertension, dyslipidemia, type $2 \mathrm{DM}$, smoking habit, SRS, SSS, stress MBF, and MPR in the model as the independent (i.e. predictive) variables, and LVEF, MFR/3, and Entropy as the dependent (i.e. outcome) variables. The independent significance of the included predictors was tested using Pillai's trace criterion with an approximate $F$ statistic. Further, the multivariate analyses were repeated including the same independent and dependent variables for patients with and without imaging evidence of a previous MI. Additionally, the effect sizes for the predictors $\left(\eta^{2}\right)$ are reported and graphically depicted.

Finally, supplementary analyses were performed in both female and hypertensive patients without a previous MI.

All statistical analyses were performed with SPSS (Released 2013. IBM SPSS Statistics for Windows, Version 22.0. Armonk, NY: IBM Corp., USA). A $P$-value of $<0.05$ was considered statistically significant.

\section{RESULTS}

Baseline characteristics, semiquantitative, quantitative perfusion, and ventricular function data are presented in Table 1. We found a high prevalence of HTN $(60 \%)$ and dyslipidemia (57\%) in the whole cohort, while nearly a quarter had undergone previous revascularization. When comparing patients with and without a previous MI, a significantly higher prevalence of type 2 DM, smokers, and dyspnea was documented in the MI group. The scan results of two representative patients are shown in Figure 1. Figure 2 shows the proportion of scans interpreted to have ischemia, MI, or both. A greater proportion of scans with a previous MI showed ischemic findings, while a smaller proportion of scans without evidence of a previous MI had ischemia.

Differences were found in clinical characteristics, perfusion (semiquantitative scores, stress $\mathrm{MBF}$, and MPR), and ventricular function parameters (LVEF, MFR/3, and Entropy) between patients with and without

Table 1. Baseline population characteristics

\begin{tabular}{|c|c|c|c|c|}
\hline Variable & $\begin{array}{c}\text { All } \\
n=248\end{array}$ & $\begin{array}{l}\text { No previous MI } \\
n=162\end{array}$ & $\begin{array}{l}\text { Previous MI } \\
n=86\end{array}$ & $P$ value \\
\hline \multicolumn{5}{|l|}{ Demographics-mean (SD) } \\
\hline Age (years) & $64(11.2)$ & $63.2(11.3)$ & $65.4(10.8)$ & .137 \\
\hline Women/men (n) & $82 / 166$ & $65 / 97$ & $17 / 69$ & .002 \\
\hline BMI $\left(\mathrm{kg} / \mathrm{m}^{2}\right)$ & $27.8(4.2)$ & $28.0(4.3)$ & $27.4(3.8)$ & .334 \\
\hline \multicolumn{5}{|l|}{ Risk factors $-\mathrm{n}(\%)$} \\
\hline Arterial hypertension & $149(60)$ & $101(62)$ & $48(56)$ & .292 \\
\hline Dyslipidemia & $141(57)$ & $90(56)$ & $51(59)$ & .540 \\
\hline Type 2 DM & $51(21)$ & $25(15)$ & $26(30)$ & .007 \\
\hline Smokers & $112(45)$ & $65(40)$ & $47(55)$ & .021 \\
\hline \multicolumn{5}{|l|}{ Cardiovascular history -n (\%) } \\
\hline Asymptomatic & $60(24)$ & $54(33)$ & $6(7)$ & $<.001$ \\
\hline Angina & $130(52)$ & $80(49)$ & $50(58)$ & .178 \\
\hline Dyspnea & $135(54)$ & $75(46)$ & $60(70)$ & $<.001$ \\
\hline Previous revascularization & $60(24)$ & $15(9)$ & $45(52)$ & $<.001$ \\
\hline \multicolumn{5}{|c|}{ Semiquantitative perfusion metrics-mean (SD) } \\
\hline SRS & $4(7)$ & $1(1)$ & $10(9)$ & $<.001$ \\
\hline SSS & $9(10)$ & $5(6)$ & $17(11)$ & $<.001$ \\
\hline SDS & $5(7)$ & $5(6)$ & 7() & .001 \\
\hline \multicolumn{5}{|c|}{ Quantitative perfusion measurements-mean (SD) } \\
\hline Rest MBF (mL/g/min) & $0.84(0.34)$ & $0.87(0.33)$ & $0.79(0.34)$ & .073 \\
\hline Stress MBF (mL/min/gr) & $1.99(0.75)$ & $2.21(0.73)$ & $1.55(0.58)$ & $<.001$ \\
\hline MPR & $2.55(0.89)$ & $2.72(0.89)$ & $2.18(0.79)$ & $<.001$ \\
\hline \multicolumn{5}{|c|}{ Ventricular function measurements-mean (SD) } \\
\hline LVEF (systolic) & $61.6(15.1)$ & $67.9(9.7)$ & $49.0(15.9)$ & $<.001$ \\
\hline MFR/3 (diastolic) & $1.12(0.38)$ & $1.21(0.35)$ & $0.91(0.36)$ & $<.001$ \\
\hline Entropy (synchrony) & $45.6(11.3)$ & $42.3(8.7)$ & $52.8(12.4)$ & $<.001$ \\
\hline
\end{tabular}

$B M I$ body mass index; $D M$ diabetes mellitus; $M I$ myocardial infarction; $M B F$ myocardial blood flow; $M P R$ myocardial perfusion reserve 
MI. There was a higher, although not significant, rest MBF in patients without MI.

The corresponding data for the subsample of women and patients with arterial hypertension can be found in Supplementary Material-Online Resource 1 and showed comparable distribution of baseline characteristics with the exception of a higher proportion of asymptomatic patients and a lower proportion of previous revascularization procedures. Also, there was a comparable rest and stress MBF in women and hypertensive patient groups.

\section{Biserial Correlations Between Dependent Variables}

We documented significant and strong correlations between systolic, diastolic, and synchrony function variables. Pearson's correlation coefficients showed significance between LVEF and MFR/3, LVEF and Entropy, and MFR/3 and Entropy. These results are depicted in the correlation matrix shown in Table 2.

\section{Multivariate Analysis of Covariance}

The multivariate analysis demonstrated that stress MBF is an independent significant predictor $(P<.001$, $\left.\eta^{2}=0.111\right)$ of the ventricular function (LVEF, MFR/3, and E), while MPR showed only a trend toward significance $(P=0.063)$. From the other clinically relevant variables included in the model, sex $(P=.003)$, age $(P=.004)$, type $2 \mathrm{DM}(P=.025)$, and SRS $(P<$ $\left..001, \eta^{2}=0.284\right)$ were also found to be significant predictors (Table 3 ).

The secondary exploratory multivariate analysis for patients without a previous MI $(n=162)$ again showed that stress MBF was significantly associated with ventricular function $\left(P=.006, \eta^{2}=0.103\right)$, while MPR was not $(P=.27)$. In this analysis, sex and BMI were also significant predictors $(P=.029$ and $P=.005$, respectively). Similar results were obtained in the analysis of patients with evidence of a previous MI: stress MBF $(P=.022)$, MPR $(P=.090)$, and SRS $(P=$ $.004)$. These results are shown in more detail in the Supplementary Material-Online Resources 2 and 3. The magnitude of the effect sizes for the primary and secondary analyses is depicted in Figure 3.

The supplementary analyses in women without a previous MI conveyed a rather small and, therefore, underpowered subsample $(n=65)$ considering the amount of relevant variables in the other analyses. As for noninfarcted patients with hypertension $(\mathrm{n}=101)$, results were similar to those in the primary and secondary analyses (Supplementary Material-Online Resource 4).
A supplementary follow-up univariate analysis divided per measurement of ventricular function can be consulted in Supplementary Material-Online Resources 5 through 7.

\section{DISCUSSION}

In the present study, we have shown that stress MBF correlates better that MPR with ventricular function in a population of patients with suspected ischemia using an integral statistical approach. By inputting both quantitative perfusion measurements (i.e. stress $\mathrm{MBF}$ and MPR) in the analyses, we have aimed to compare their relative importance based on their independent association with ventricular function.

In the first part of the study, we documented differences between important subgroups concerning the presence or the absence of a previous MI. Results have confirmed important differences in ventricular perfusion and several components of ventricular function as well as in clinical profile between these two groups of patients. Patients with evidence of a previous MI had a higher prevalence of type $2 \mathrm{DM}$ which adds to their prognostic profile. Interestingly, they were more symptomatic and showed slightly more ischemic burden overall. As expected, a worse ventricular function profile was found. However, not only scar but also perfusion seems to play a role in their functional status.

A close statistical relationship between systolic, diastolic, and synchronic ventricular function was observed. In the clinical scenario, functional measurements may individually serve at different points of the spectrum of severity of CAD. Nonetheless, they also provide, in compound, a proxy for the overall status of ventricular function since in reality they are probably not modified independently. Still, further research is needed to define the optimal measurements of diastolic function and synchrony, as well as their particular normal values in different clinical scenarios evaluated with PET imaging.

Next, we documented that stress MBF is consistently better correlated to ventricular function than MPR as evidenced by their significance values and moderate effect sizes in the multivariate models (Figure 3), independently from the extent and severity of a previous MI (as measured by SRS) or other clinically relevant variables. Nonetheless, it became clear in the primary analysis that the greatest influence on cardiac function patent from our analyses was the presence of a previous MI.

The significant association of stress MBF with ventricular function was sustained in the supplementary analysis of patients with hypertension and without previous MI. These results suggest a greater relevance of stress MBF, rather than MPR, in the profile of patients who may benefit from its evaluation in routine clinical 


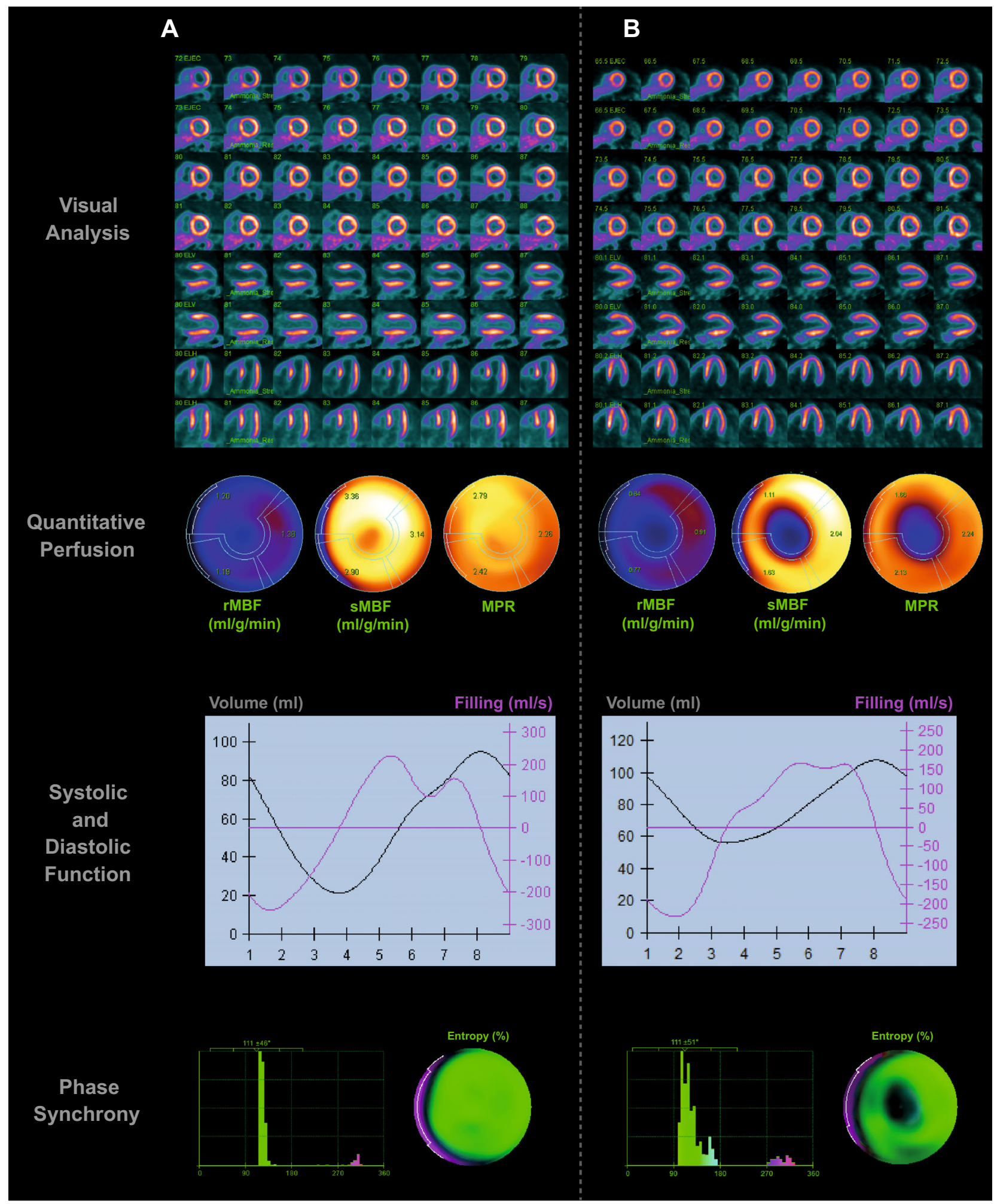

Figure 1. Representative cases from the spectrum of patients in the study. A shows the perfusion and functional results of a 55-year-old female with a low-likelihood cardiovascular risk, no quantitative perfusion abnormalities: $\mathrm{MPR}=2.58, \mathrm{sMBF}=2.22 \mathrm{~mL} / \mathrm{g} / \mathrm{min}$, and preserved systolic, diastolic, and synchronic function: $\mathrm{LVEF}=75 \%, \mathrm{MFR} / 3=1.25, \mathrm{E}=34 \%$. B Shows a 64-year-old male with history of a previous distal anterior and apical MI with residual anteroapical ischemia: MPR $=1.88, \mathrm{sMBF}=1.43$ and diminished systolic, diastolic, and synchronic function: $\mathrm{LVEF} 47 \%$, $\mathrm{MFR} / 3=0.55, \mathrm{E}=61 \%$. 


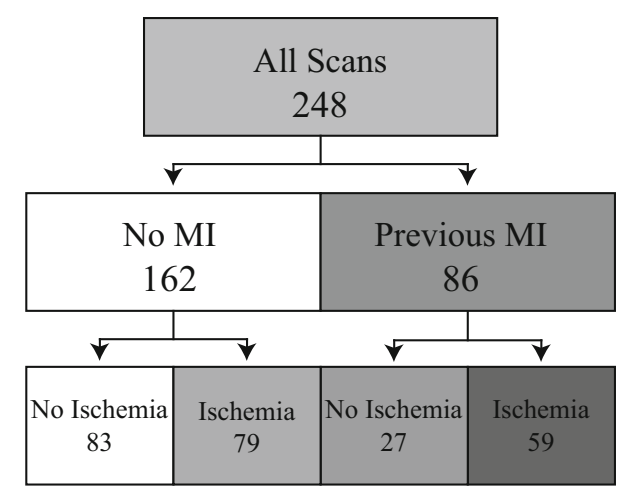

Figure 2. Division of scans according to their interpretation.

Table 2. Biserial correlations between dependent variables analyzed through Pearson's correlation coefficient

\begin{tabular}{llll}
\hline & LVEF & MFR/3 & \multicolumn{1}{c}{$\boldsymbol{E}$} \\
\hline LVEF & 1 & $0.612^{*}$ & $-0.698^{*}$ \\
MFR/3 & $0.612^{*}$ & 1 & $-0.563^{*}$ \\
$E$ & $-0.698^{*}$ & $-0.563^{*}$ & 1 \\
\hline
\end{tabular}

E entropy; $L V E F$ left ventricular ejection fraction; $M F R / 3$ mean filling rate during the first third of the diastole.

${ }^{*} P<0.001$ practice. Moreover, considering the use of stress MBF as an alternative to MPR may be of benefit considering patient safety for the evaluation of ischemic burden. In this sense, acquisition times and radiation doses can be reduced $^{26}$ through "stress-only" protocols. This, however, may not be implemented in patients with history of a previous MI, where rest defects should be evaluated.

We have found other significant predictors of ventricular function, although not as strong as stress MBF, namely: sex, age, and type 2 DM. These results support previous studies describing sex-based systolic function differences, a progressive decline of diastolic function associated with age, ${ }^{27}$ and the vascular-function-independent effect of type $2 \mathrm{DM}$ on myocardial contraction. $^{28}$

The clinical use of quantitative perfusion goes beyond diagnosis. Risk stratification and prediction of CAD-related outcomes are of major interest in noninvasive cardiac imaging due to the major burden that cardiovascular disease represents. ${ }^{29}$ Notably, our results support the potential utility of stress MBF over MPR in two main aspects. First, the role of stress MBF in patients that have not suffered a major event, such as MI, may be primarily diagnostic, as it has been proposed in studies focused on the detection of anatomically significant CAD. ${ }^{8}$ Then, stress MBF may be sensitively hampered and additionally provide information on the functional consequences of myocardial ischemia. As

Table 3. Multivariate model results for significant predictors of the integrative ventricular function, $\mathrm{n}=248$

\begin{tabular}{|c|c|c|c|c|c|c|}
\hline \multicolumn{7}{|c|}{ Multivariate analysis of covariance } \\
\hline $\begin{array}{c}\text { Dependent } \\
\text { variables }\end{array}$ & $\begin{array}{l}\text { Independent } \\
\text { variables }\end{array}$ & $\begin{array}{l}\text { Pillai's Trace Value } \\
\left.\text { (and } \eta^{2}\right)\end{array}$ & $\mathbf{F}$ & $\begin{array}{l}\text { Hypothesis } \\
\text { df }\end{array}$ & $\begin{array}{l}\text { Error } \\
\text { dff }\end{array}$ & $\begin{array}{c}P \\
\text { value }\end{array}$ \\
\hline LVEF & Intercept & 0.630 & 98.705 & 3.0 & 174.0 & $<.001$ \\
\hline MFR/3 & Sex & 0.077 & 4.861 & & & $.003 *$ \\
\hline \multirow[t]{10}{*}{ Entropy } & Age & 0.073 & 4.586 & & & $.004^{*}$ \\
\hline & Hypertension & 0.015 & 0.870 & & & .458 \\
\hline & Dyslipidemia & 0.010 & 0.606 & & & .612 \\
\hline & Type 2 DM & 0.052 & 3.187 & & & $.025^{*}$ \\
\hline & Smoking & 0.033 & 1.967 & & & .121 \\
\hline & BMI & 0.031 & 1.841 & & & .141 \\
\hline & SRS & 0.284 & 22.966 & & & $<.001$ * \\
\hline & SSS & 0.028 & 1.695 & & & .170 \\
\hline & Stress MBF & 0.111 & 7.253 & & & $<.001$ * \\
\hline & MPR & 0.041 & 2.474 & & & .063 \\
\hline
\end{tabular}

$B M I$ body mass index; $d f$ degrees of freedom; $D M$ diabetes mellitus; $L V E F$ left ventricular ejection fraction; $M B F$ myocardial blood flow; MFR $/ 3$ mean filling rate during the first third of the diastole; MPR myocardial perfusion reserve; SRS summed rest score; SSS summed stress score

* Significant $P$ value 


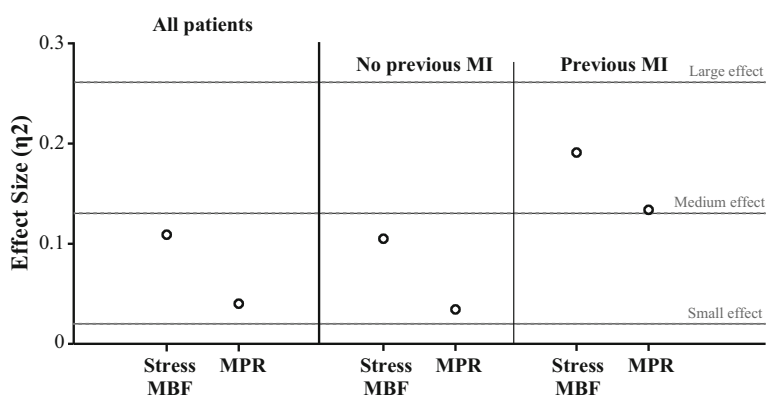

Figure 3. Point graph with the effect sizes of stress MBF and MPR estimated by the multivariate analyses.

already mentioned, this application can potentially prevent unnecessary radiation exposure if a resting phase scan is spared. Second, in the case of patients with a previous MI, the main role of stress MBF may be prognostic, ${ }^{9,10}$ given its relevance in influencing residual ventricular function. Future research on condition-adjusted cut-offs and on the prognostic performance of stress MBF for specific outcomes, such as heart failure, is warranted.

A previous report from our group ${ }^{30}$ has addressed the relationship between quantitative myocardial perfusion and systolic function. However, such study was performed in a highly restricted population including only patients with previous MI and varying degrees of systolic dysfunction, and it was mostly focused on MPR rather than stress MBF. The present study extends our knowledge concerning the relationships of stress MBF by demonstrating a stronger association with ventricular function in comparison with MPR, and in a wider population of patients referred for cardiac PET imaging.

We believe that further investigation will optimize the application of cardiac PET by exploring the clinical value of combined perfusion-function measurements which may offer more integral gauges of cardiac health.

\section{NEW KNOWLEDGE GAINED}

This study demonstrated that peak-stress MBF correlates with ventricular function (composed by measures of systolic, diastolic, and synchronic function) better than MPR and independently from relevant covariates. Additionally, the present work showed that the described greater correlation of stress MBF with ventricular function is sustained in the patients with and without a previous MI and in noninfarcted patients with arterial hypertension.

\section{LIMITATIONS}

One of the characteristics of our study is that we have approached a fairly heterogeneous population.
Although this potentially yields difficulty in addressing multivariate relations, we believe that this approach rather provides a broad view of these complex relations in the setting of the day-to-day reality of specialized cardiovascular imaging centers. Another potential limitation of our study, especially for the assessment of rather novel diastolic function parameters such as MFR/ 3 , is that the analysis of the gated datasets was performed with a binning of 8 frames per cardiac cycle. Although this may not be optimal, there are a number of studies which have demonstrated that the obtained assessment and derivation of the time-volume curves from 8 frames is comparable to SPECT reconstructions made using 64 frames. In the future, complementary echocardiographic evaluation can be useful. Yet, another limitation is the lack of regional perfusion analysis. Although of interest, we aimed to account for the global perfusion status and its influence on the global ventricular function. Therefore, the influence of specific regional measures cannot be evaluated from this study alone.

\section{CONCLUSIONS}

Stress MBF is better correlated with ventricular function than MPR, as evaluated by Nitrogen-13 ammonia PET, independently from other relevant cardiovascular risk factors and clinical covariates. This relationship between coronary vasodilatory capacity and ventricular function is sustained across groups with and without a previous MI. Further research in the clinical utility of PET evaluation of stress MBF and its prognostic value is warranted.

\section{Acknowledgements}

We would like to acknowledge the substantial and assertive advice of Dr. David Vállez García in the methodological review of the present work. Also, we would like to declare the invaluable labor and technical assistance of the medical students and the technical staff (Jorge Albarrán and Andrés Sanabria) from the PET Unit in Mexico City.

\section{Disclosure}

The authors have no conflicts of interest to declare.

\section{Open Access}

This article is distributed under the terms of the Creative Commons Attribution 4.0 International License ( http://creativecommons.org/licenses/by/4.0/), which permits unrestricted use, distribution, and reproduction in any medium, provided you give appropriate credit to the original author(s) and the source, provide a link to the Creative Commons license, and indicate if changes were made. 


\section{References}

1. Qayyum AA, Kastrup J. Measuring myocardial perfusion: The role of PET, MRI and CT. Clin Radiol [Internet]. The Royal College of Radiologists; 2015;70(6):576-84. http://linkinghub. elsevier.com/retrieve/pii/S0009926015000215

2. Hajjiri MM, Leavitt MB, Zheng H, Spooner AE, Fischman AJ, Gewirtz H. Comparison of positron emission tomography measurement of adenosine-stimulated absolute myocardial blood flow versus relative myocardial tracer content for physiological assessment of coronary artery stenosis severity and location. JACC Cardiovasc Imaging [Internet]. 2009;2(6):751-8. http://www. embase.com/search/results?subaction=viewrecord\&from=export\& id=L354675694

3. Kajander SA, Joutsiniemi E, Saraste M, Pietilä M, Ukkonen H, Saraste A, et al. Clinical value of absolute quantification of myocardial perfusion with ${ }^{15} \mathrm{O}$-water in coronary artery disease. Circ Cardiovasc Imaging. 2011;4(6):678-84.

4. Arsanjani R, Xu Y, Hayes SW, Fish M, Lemley M, Gerlach J, et al. Comparison of fully automated computer analysis and visual scoring for detection of coronary artery disease from myocardial perfusion SPECT in a large population. J Nucl Med [Internet]. 2013;54(2):221-8. http://jnm.snmjournals.org/cgi/doi/10.2967/jn umed.112.108969. Accessed 1 Feb 2013

5. Waller AH, Blankstein R, Kwong RY, Di Carli MF. Myocardial blood flow quantification for evaluation of coronary artery disease by positron emission tomography, cardiac magnetic resonance imaging, and computed tomography. Curr Cardiol Rep [Internet]. 2014;16(5):483. http://www.ncbi.nlm.nih.gov/pubmed/24718671

6. Schindler TH, Schelbert HR, Quercioli A, Dilsizian V. Cardiac PET imaging for the detection and monitoring of coronary artery disease and microvascular health. JACC Cardiovasc Imaging [Internet]. Elsevier Inc.; 2010;3(6):623-40. http://www.ncbi.nlm. nih.gov/pubmed/20541718

7. Taqueti VR, Hachamovitch R, Murthy VL, Naya M, Foster CR, Hainer J, et al. Global coronary flow reserve is associated with adverse cardiovascular events independently of luminal angiographic severity and modifies the effect of early revascularization. Circulation. 2015;131(1):19-27.

8. Joutsiniemi E, Saraste A, Pietila M, Maki M, Kajander S, Ukkonen $\mathrm{H}$, et al. Absolute flow or myocardial flow reserve for the detection of significant coronary artery disease? Eur Hear J - Cardiovasc Imaging [Internet]. 2014;15(6):659-65. http://ehjcimaging. oxfordjournals.org/cgi/doi/10.1093/ehjci/jet274. Accessed 1 Jun 2014

9. Farhad H, Dunet V, Bachelard K, Allenbach G, Kaufmann PA, Prior JO. Added prognostic value of myocardial blood flow quantitation in rubidium-82 positron emission tomography imaging. Eur Hear J - Cardiovasc Imaging [Internet]. 2013;14(12): 1203-10. Go to ISI://WOS:000326966100015. Accessed 1 Dec 2013

10. Murthy VL, Naya M, Foster CR, Hainer J, Gaber M, Di Carli G, et al. Improved cardiac risk assessment with noninvasive measures of coronary flow reserve. Circulation [Internet]. 2011;124(20): 2215-24. http://www.ncbi.nlm.nih.gov/pubmed/22007073. Accessed 15 Nov 2011

11. Hickey KT, Sciacca RR, Bokhari S, Rodriguez O, Chou R-L, Faber TL, et al. Assessment of Cardiac Wall Motion and Ejection Fraction With Gated PET Using N-13 Ammonia. Clin Nucl Med [Internet]. 2004;29(4):243-8. http://content.wkhealth.com/link back/openurl?sid=WKPTLP:landingpage $\&$ an $=00003072-2004040$ 00-00003

12. Hattori N, Bengel FM, Mehilli J, Odaka K, Ishii K, Schwaiger M, et al. Global and regional functional measurements with gated FDG PET in comparison with left ventriculography. Eur J Nucl
Med [Internet]. 2001;28(2):221-9. http://link.springer.com/10. 1007/s002590000441. Accessed 7 Feb 2001

13. Schaefer WM, Lipke CSA, Standke D, Kühl HP, Nowak B, Kaiser $\mathrm{H}-\mathrm{J}$, et al. Quantification of left ventricular volumes and ejection fraction from gated ${ }^{99 \mathrm{~m}}$ Tc-MIBI SPECT: MRI validation and comparison of the Emory Cardiac Tool Box with QGS and 4DMSPECT. J Nucl Med [Internet]. 2005;46(8):1256-63. http://www.ncbi.nlm.nih.gov/pubmed/16085580

14. O'Connell JW, Schreck C, Moles M, Badwar N, DeMarco T, Olgin J, et al. A unique method by which to quantitate synchrony with equilibrium radionuclide angiography. J Nucl Cardiol [Internet]. 2005;12(4):441-50. http://link.springer.com/10.1016/j. nuclcard.2005.05.006

15. Chen J, Faber TL, Cooke CD, Garcia E V. Temporal resolution of multiharmonic phase analysis of ECG-gated myocardial perfusion SPECT studies. J Nucl Cardiol [Internet]. 2008;15(3):383-91. http://link.springer.com/10.1016/j.nuclcard.2008.02.014

16. Van Tosh A, Votaw JR, Reichek N, Palestro CJ, Nichols KJ. The relationship between ischemia-induced left ventricular dysfunction, coronary flow reserve, and coronary steal on regadenoson stress-gated $82 \mathrm{Rb}$ PET myocardial perfusion imaging. J Nucl Cardiol [Internet]. 2013;20(6):1060-8. http://link.springer.com/ 10.1007/s12350-013-9784-1. Accessed 4 Dec 2013

17. Dilsizian V, Bacharach SL, Beanlands RS, Bergmann SR, Delbeke D, Gropler RJ, et al. PET myocardial perfusion and metabolism clinical imaging. J Nucl Cardiol [Internet]. 2009;16(4):651-651. http://link.springer.com/10.1007/s12350-009-9094-9. Accessed 4 Aug 2009

18. Slomka PJ, Alexanderson E, Jácome R, Jiménez M, Romero E, Meave A, et al. Comparison of clinical tools for measurements of regional stress and rest myocardial blood flow assessed with ${ }^{13} \mathrm{~N}$ ammonia PET/CT. J Nucl Med [Internet]. 2012;53(2):171-81. http://www.ncbi.nlm.nih.gov/pubmed/22228795

19. Choi Y, Huang SC, Hawkins RA, Kuhle WG, Dahlbom M, Hoh $\mathrm{CK}$, et al. A simplified method for quantification of myocardial blood flow using nitrogen-13-ammonia and dynamic PET. J Nucl Med [Internet]. 1993;34(3):488-97. http://www.ncbi.nlm.nih.gov/ pubmed/8280197

20. Kaufmann PA, Gnecchi-Ruscone T, Yap JT, Rimoldi O, Camici PG. Assessment of the reproducibility of baseline and hyperemic myocardial blood flow measurements with ${ }^{15} \mathrm{O}$-labeled water and PET. J Nucl Med [Internet]. 1999;40(11):1848-56. http://www. ncbi.nlm.nih.gov/pubmed/10565780

21. Sharir T, Germano G, Kavanagh PB, Lai S, Cohen I, Lewin HC, et al. Incremental prognostic value of post-stress left ventricular ejection fraction and volume by gated myocardial perfusion single photon emission computed tomography. Circulation. 1999; 100(10): 1035-42.

22. Germano G, Kiat H, Kavanagh PB, Moriel M, Mazzanti M, Su HT, et al. Automatic quantification of ejection fraction from gated myocardial perfusion SPECT. J Nucl Med. 1995;36(11):2138-47.

23. Higuchi T, Nakajima K, Taki J, Kinuya S, Bunko H, Tonami N. Assessment of left ventricular systolic and diastolic function based on the edge detection method with myocardial ECG-gated SPET. Eur J Nucl Med Mol Imaging [Internet]. 2001;28(10):1512-6. http://link.springer.com/10.1007/s002590100619. Accessed 1 Oct 2001

24. Nakajima K, Taki J, Kawano M, Higuchi T, Sato S, Nishijima C, et al. Diastolic dysfunction in patients with systemic sclerosis detected by gated myocardial perfusion SPECT: An early sign of cardiac involvement. J Nucl Med [Internet]. 2001;42(2):183-8. http://www.ncbi.nlm.nih.gov/pubmed/11216514

25. Uebleis C, Ulbrich M, Tegtmeyer R, Schuessler F, Haserueck N, Siebermair J, et al. Electrocardiogram-Gated 18F-FDG PET/CT 
Hybrid Imaging in Patients with Unsatisfactory Response to Cardiac Resynchronization Therapy: Initial Clinical Results. J Nucl Med [Internet]. 2011;52(1):67-71. http://jnm.snmjournals.org/ cgi/doi/10.2967/jnumed.110.078709. Accessed 1 Jan 2011

26. Schindler TH, Dilsizian V. PET-Determined Hyperemic Myocardial Blood Flow. J Am Coll Cardiol [Internet]. Elsevier Inc; 2014;64(14):1476-8. http://linkinghub.elsevier.com/retrieve/pii/S 0735109714057106

27. Abinader EG, Sharif DS. Age-associated changes in left ventricular diastolic performance during isometric stress. Am J Cardiol [Internet]. 1993;71(7):629. http://www.ncbi.nlm.nih.gov/pubmed/ 8438761. Accessed 1 Mar 1993

28. Juárez-Orozco LE, van der Zant FM, Slart RHJA, Lazarenko S V., Alexanderson E, Tio RA, et al. Type 2 diabetes mellitus correlates with systolic function during myocardial stress perfusion scanning with Nitrogen-13 ammonia PET. J Nucl Cardiol [Internet]. Available from: http://link.springer.com/10.1007/s12350-0160482-7. Accessed 15 Apr 2016

29. Mathers CD, Loncar D. Projections of global mortality and burden of disease from 2002 to 2030. PLoS Med [Internet]. 2006 Nov;3(11):e442. http://www.ncbi.nlm.nih.gov/pubmed/17132052

30. Juárez-Orozco LE, Glauche J, Alexanderson E, Zeebregts CJ, Boersma HH, Glaudemans AWJM, et al. Myocardial perfusion reserve in spared myocardium: correlation with infarct size and left ventricular ejection fraction. Eur J Nucl Med Mol Imaging [Internet]. 2013 Aug 4;40(8):1148-54. http://www.ncbi.nlm.nih. gov/pubmed/23553081 